\title{
PIKfyve Sensitivity of hERG Channels
}

\author{
Tatsiana Pakladok Ahmad Almilaji Carlos Munoz Ioana Alesutan Florian Lang
}

Department of Physiology, University of Tübingen, Tübingen

\author{
Key Words

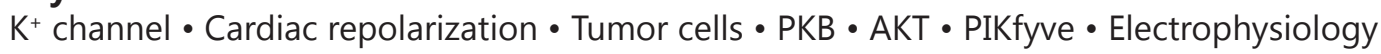

\begin{abstract}
Background/Aims: Human ether-a-go-go ( $h E R G$ ) channels contribute to cardiac repolarization and participate in the regulation of tumor cell proliferation. Mutations in hERG channels may cause long QT syndrome and sudden cardiac death due to ventricular arrhythmias. HERG channel activity is up-regulated by the serum- and glucocorticoid-inducible kinase isoforms SGK1 and SGK3. Related kinases are protein kinase B (PKB/Akt) isoforms. SGK's and PKB/ Akt's activate phosphatidylinositol-3-phosphate-5-kinase PIKfyve, which in turn up-regulates several carriers and channels. An effect of PIKfyve on hERG channels, has, however, never been shown. The present study thus explored the putative influence of PIKfyve on hERG channel expression and activity. Methods: hERG channels were expressed in Xenopus oocytes with or without PIKfyve and/or PKB, expression of endogenous and injected hERG quantified by RT$P C R$, and hERG channel activity determined utilizing dual electrode voltage clamp. Moreover, hERG protein abundance in the cell membrane was visualized utilizing specific antibody binding and subsequent confocal microscopy and quantified by chemiluminescence. Results: Coexpression of wild type PIKfyve increased hERG channel activity in hERG-expressing Xenopus oocytes. hERG channel activity was further increased by coexpression of PKB, an effect augmented by additional coexpression of PIKfyve, but not by additional coexpression of PKB/Akt-resistant PIKfyve mutant PIKfyve ${ }^{\mathrm{S} 318 \mathrm{~A}}$. Coexpression of PIKfyve increased hERG channel protein abundance in the cell membrane. Inhibition of hERG channel insertion into the cell membrane by Brefeldin A $(5 \mu \mathrm{M})$ resulted in a decline of current, which was similar in Xenopus oocytes expressing hERG together with PIKfyve and in Xenopus oocytes expressing hERG alone. Conclusion: hERG is up-regulated by PIKfyve, which is in turn activated by PKB/ Akt.
\end{abstract}

Copyright $@ 2013$ S. Karger AG, Basel 


\section{Cellular Physiology Cell Physiol Biochem 2013;31:785-794 \begin{tabular}{l|l} 
and Biochemistry Published onIIne: Vlay 31, 2013 & $\begin{array}{l}\text { C 2013 S. Karger AG, Basel } \\
\text { www.karger.com/cpb }\end{array}$ \\
\hline
\end{tabular} \\ Pakladok/Almilaji/Munoz/Alesutan/Lang: PIKfyve-Regulated hERG Channels}

\section{Introduction}

The human ether-a-go-go (hERG) channels contribute to cardiac repolarization [1, 2]. Moreover, hERG channels are expressed in several tumor cells and contribute to the regulation of cell proliferation [3-5]. Owing to the role of hERG in tumor cells, hERG inhibitors have been considered for the treatment of malignancy [4-7].

hERG channels are up-regulated by the serum-and glucocorticoid-inducible kinase isoform SGK1 and SGK3 [8, 9]. SGK isoforms [10] and the related PKB/Akt isoforms [11] regulate carrier proteins in part by phosphorylation and activation of phosphatidylinositol3-phosphate-5-kinase PIKfyve [11-14], also named PIPkIII [15], or PIP5K [16], which is the mammalian ortholog of the yeast Fab1p [17]. Mediated by its FYVE finger domain [18, 19], PIKfyve binds to PtdIns3P localized in membranes of late endocytic compartments [20]. PIKfyve converts PtdIns3P into PtdIns(3,5) $P_{2}$ thus regulating a variety of cell functions [21]. PIKfyve participates in the regulation of retrograde trafficking from the early endosome to the trans-Golgi network (TGN) [22] and thus modifies cell membrane protein trafficking [19, 23-26]. By this means, PIKfyve regulates glucose transporters [11-13], creatine transporter [27], glutamate transporters [28-30], glutamate receptors [31], $\mathrm{Ca}^{2+}$ channels [32], $\mathrm{Cl}^{-}$ channels [30,33] and inward rectifier potassium channels [34].

In view of the effects of SGK1 and SGK3 on hERG channel activity and considering the role of PIKfyve in the actions of PKB/Akt and SGK isoforms, we hypothesized that PIKfyve participates in the regulation of hERG. In order to test this hypothesis, hERG was expressed in Xenopus oocytes with or without the expression of PIKfyve, and hERG channel function was determined utilizing dual-electrode voltage clamp and hERG channel protein abundance in the cell membrane by chemiluminescence as well as immunocytochemistry with subsequent confocal microscopy.

\section{Materials and Methods}

For generation of cRNA [35], constructs were used encoding hERG channels [8], hERG-HA containing an extracellular hemagglutinin epitope [36], human wild type PKB/AKT1 [37, 38], wild-type PIKfyve [28], and PKB resistant PIKfyve ${ }^{\mathrm{S318A}}$ [28]. The cRNA was generated as described previously [39, 40].

To determine hERG transcripts levels, total RNA was isolated from Xenopus oocytes with or without injection of hERG cRNA by using Trifast Reagent (Peqlab Biotechnologie GmbH, Germany) according to the manufacturer's instructions. Reverse transcription of $2 \mu \mathrm{g}$ RNA was performed using oligo $(\mathrm{dT})_{12-18}$ primers (Invitrogen, Life Technologies GmbH, Germany) and SuperScriptIII Reverse Transcriptase (Invitrogen, Life Technologies GmbH, Germany). cDNA samples were treated with RNaseH (Invitrogen, Life Technologies GmbH, Germany). Quantitative real-time PCR was performed with the iCycler iQ ${ }^{\mathrm{TM}}$ Real-Time PCR Detection System (Bio-Rad Laboratories GmbH, Germany) and $\mathrm{iQ}^{\mathrm{TM}}$ Sybr Green Supermix (Bio-Rad Laboratories, $\mathrm{GmbH}$, Germany) according to the manufacturer's instructions. The following primers were used (5'->3' orientation): human ERG fw: CAACCTGGGCGACCAGATAG; human ERG rev: GGTGTTGGGAGAGACGTTGC; Xenopus laevis Gapdh fw: GACCTGCCGCCTGCAGAAG; Xenopus laevis Gapdh rev: GACTAGCAGGATGGGCGAC. The specificity of the PCR products was confirmed by analysis of the melting curves. All PCRs were performed in duplicate, and mRNA fold changes were calculated by the $2^{-\Delta \Delta C t}$ method using Gapdh as internal reference. Averaging and statistical tests were carried out with the logarithmic values of transcripts levels.

For voltage clamp analysis, Xenopus oocytes were prepared as previously described [37]. Where indicated oocytes were injected with water or $10 \mathrm{ng}$ cRNA encoding PKB, PIKfyve and/or PIKfyve ${ }^{5318 A}$ and later on the same day with $10 \mathrm{ng}$ cRNA encoding hERG. Standard two electrode voltage clamp recordings were performed 3 days after hERG injection $[41,42]$. The oocytes were maintained at $17^{\circ} \mathrm{C}$ in a solution containing $88.5 \mathrm{mM} \mathrm{NaCl}, 2 \mathrm{mM} \mathrm{KCl}, 1 \mathrm{mM} \mathrm{MgC1} 1_{2}, 1.8 \mathrm{mM} \mathrm{CaC1}_{2}, 5 \mathrm{mM}$ HEPES, tretracycline (Sigma, $0.11 \mathrm{mM}$ ), ciprofloxacin (Sigma, $4 \mu \mathrm{M}$ ), gentamycin (refobacin (C) $0.2 \mathrm{mM}$ ) and theophylin (euphylong (C), $0.5 \mathrm{mM}$ ) as well as sodium pyruvate (Sigma, $5 \mathrm{mM}$ ) were added to the ND96, $\mathrm{pH}$ was adjusted to 7.5 by addition of $\mathrm{NaOH}$. The control superfusate contained $96 \mathrm{mM} \mathrm{NaCl}, 2 \mathrm{mM} \mathrm{KCl}, 1.8 \mathrm{mM} \mathrm{CaCl}_{2}, 1 \mathrm{mM} \mathrm{MgCl}_{2}$ and 5 mM HEPES, pH was adjusted to 7.4 by addition of $\mathrm{NaOH}$ [43]. 


\section{Cellular Physiology Cell Physiol Biochem 2013;31:785-794

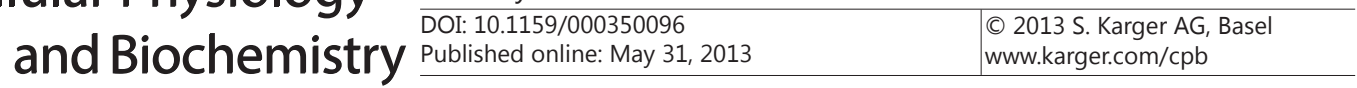 \\ Pakladok/Almilaji/Munoz/Alesutan/Lang: PIKfyve-Regulated hERG Channels}

Pipettes were filled with $3 \mathrm{M} \mathrm{KCl}$ and had resistances of 0.3-3.0 $\mathrm{M} \Omega$. Experiments were performed with a Geneclamp 500B amplifier (Axon Instruments, Union City, CA, USA) and a Digidata 1322A interface (Axon Instruments, Union City, CA, USA). Data acquisition was achieved with pCLAMP 9.2 (Axon Instruments, Union City, CA, USA) [44]. For data analysis, leak currents were subtracted. Leak currents were estimated from the tail current measured after the preconditioning prepulse to $-80 \mathrm{mV}$. To discriminate between alterations of insertion and retrieval of hERG channel protein from the plasma membrane, the insertion was inhibited by Brefeldin A [45], where indicated. In those experiments, the oocytes were preincubated for 24 and 48 hours before measurement in the presence of Brefeldin A (Sigma, Schnelldorf, Germany) at a concentration of $5 \mu \mathrm{M}[46]$.

To visualize the hERG-HA protein abundance in the cell membrane, immunocytochemistry was performed. After 4\% paraformaldehyde/PBS fixation for $2 \mathrm{~h}$, oocytes were cryoprotected in $30 \%$ sucrose, frozen in mounting medium, and placed on a cryostat. Sections were collected at a thickness of $8 \mu \mathrm{m}$ on coated slides and stored at $-20^{\circ} \mathrm{C}$. For immunostaining, sections were thawed at room temperature, fixed in acetone/methanol (1:1) for $15 \mathrm{~min}$ at room temperature, washed in PBS and blocked for 1 hour in 5\% bovine serum albumin/PBS. Sections were incubated overnight at $4^{\circ} \mathrm{C}$ with the primary $1 \mu \mathrm{g} / \mathrm{mL}$ rat monoclonal anti-HA antibody (clone 3 F10, Roche, Mannheim, Germany). Binding of primary antibody was visualised with fluorescence-labeled secondary Alexa Fluor 488 goat anti-rat IgG (1:200, Invitrogen, UK) for $1 \mathrm{~h}$ at room temperature. The slides were mounted with Pro Long Gold antifade reagent (Invitrogen, UK). Images were taken on Zeiss LSM5 EXCITER confocal laser scanning microscope (Carl Zeiss MicroImaging GmbH, Germany) with A-Plan 40x/1.2W. Brightness and contrast settings were kept constant during imaging of all oocytes in each injection series. Due to autofluorescence of the oocyte yolk, unspecific immunofluorescence was observed inside the oocytes.

To determine hERG-HA cell surface expression by chemiluminescence [28], the oocytes were incubated with $1 \mu \mathrm{g} / \mathrm{mL}$ primary rat monoclonal anti-HA antibody (clone 3 F10, Roche, Mannheim, Germany) and subsequently with secondary HRP-conjugated goat anti-rat IgG antibody (1:1000, Cell Signaling Technology, MA, USA). Individual oocytes were placed in 96 well plates with $20 \mu \mathrm{l}$ of SuperSignal ELISA Femto Maximum Sensitivity Substrate (Pierce, Rockford, IL, USA) and chemiluminescence of single oocytes was quantified in a luminometer (Walter Wallac 2 plate reader, Perkin Elmer, Juegesheim, Germany) by integrating the signal

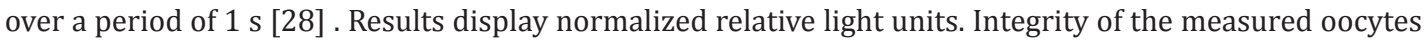
was assessed by visual control after the measurement to avoid unspecific light signals from the cytosol.

Data are provided as arithmetic means \pm SEM or geometric mean \pm SEM as indicated in the figure legends; $\mathrm{n}$ represents the number of oocytes investigated. All oocyte experiments were repeated with at least three batches of oocytes; in all repetitions, qualitatively similar data were obtained. All data were tested for significance by using one way ANOVA with Tukey's post-hoc test and $\mathrm{p}<0.05$ was considered statistically significant.

\section{Results}

The present study explored whether the phosphatidylinositol-3-phosphate-5-kinase PIKfyve influences the function of human ether-a-go-go (hERG) channels. To this end, cRNA encoding hERG was injected either with or without cRNA encoding PIKfyve. Quantitative RTPCR was employed to test for hERG expression. As illustrated in Fig. 1A, ERG transcript levels are detectable in Xenopus oocytes injected with water or with PIKfyve cRNA alone. Thus, Xenopus oocytes express low levels of endogenous ERG channels. Coinjection of hERG cRNA significantly increased hERG transcripts levels in Xenopus oocytes. HERG-mediated current was determined utilizing dual electrode voltage clamp. The channel activity was analyzed by depolarization from $-80 \mathrm{mV}$ holding potential to different voltages followed by a $500 \mathrm{~ms}$ pulse to $-60 \mathrm{mV}$. In Xenopus oocytes expressing hERG but not in water-injected oocytes or PIKfyve-injected oocytes the maneuver resulted in outward tail currents (Fig. 1B,C). In hERGexpressing Xenopus oocytes, the additional expression of wild type PIKfyve significantly increased the tail current (Fig. 1B,C). Fig. 1D illustrates the current-voltage relationship of hERG currents with or without coexpression of wild type PIKfyve. The amplitude of the peak tail current was plotted as a function of the preceding test potential. As illustrated in Fig. 1D, 


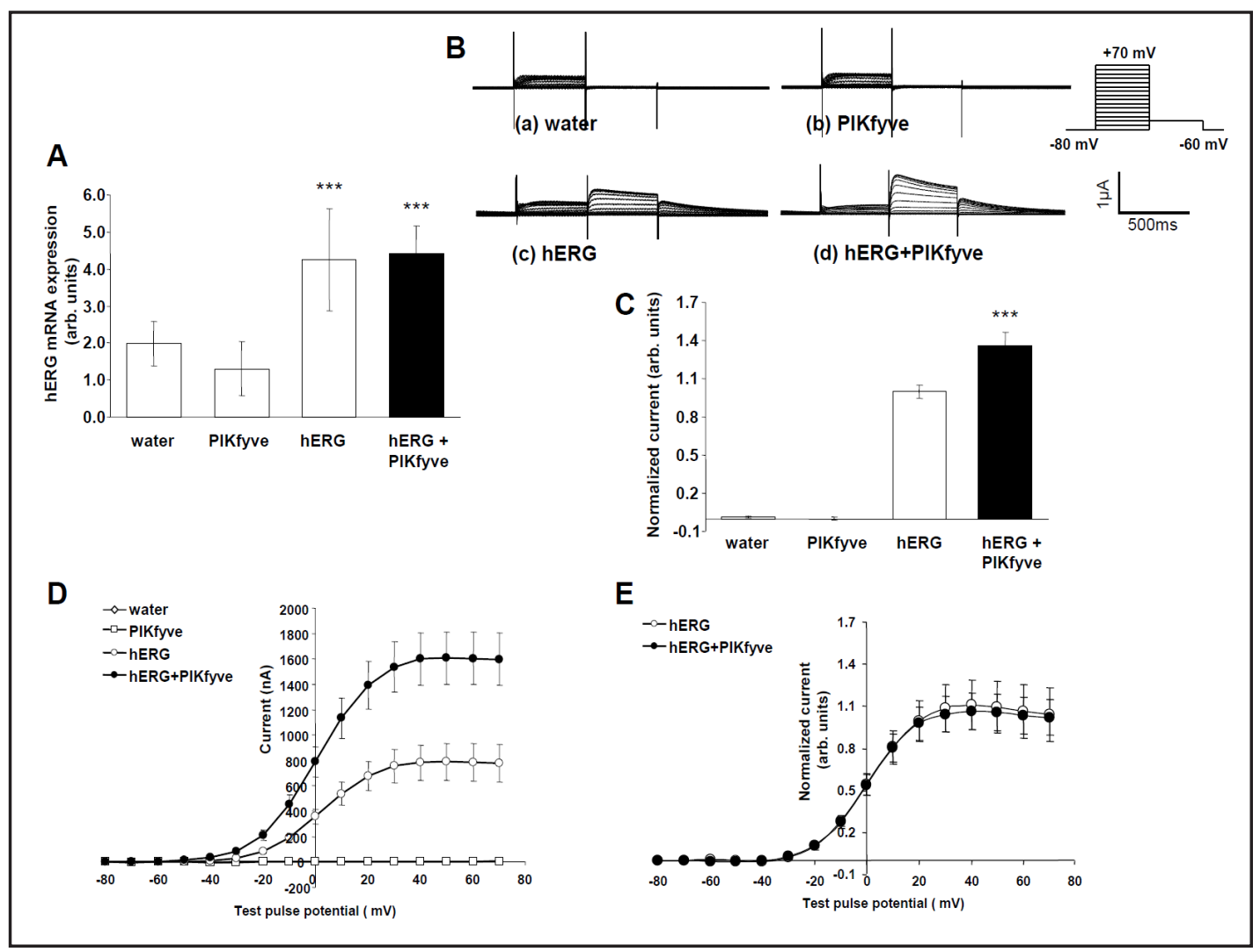

Fig. 1. Coexpression of PIKfyve increased hERG current in Xenopus oocytes. A. Geometric means \pm SEM $(n=5)$ of hERG transcripts levels in Xenopus oocytes injected with water, with cRNA encoding PIKfyve alone, with cRNA encoding hERG alone, or with cRNA encoding hERG and PIKfyve. $* * *(p<0.001)$ indicates statistically significant difference from Xenopus oocytes injected with water. B. Original tracings recorded in Xenopus oocytes injected with water (a), with cRNA encoding PIKfyve alone (b), with cRNA encoding hERG alone (c) or with cRNA encoding hERG together with PIKfyve (d). The Xenopus oocytes were depolarized from $-80 \mathrm{mV}$ holding potential to different voltages followed by a $500 \mathrm{~ms}$ pulse to $-60 \mathrm{mV}$ evoking outward tail currents. C. Arithmetic means \pm SEM $(n=13-20)$ of the normalized outward tail current following a depolarization to $+70 \mathrm{mV}$, recorded in Xenopus oocytes injected with water, with cRNA encoding PIKfyve alone, with cRNA encoding hERG alone, or with cRNA encoding hERG and PIKfyve. ${ }^{* * *}(\mathrm{p}<0.001)$ indicates statistically significant difference from Xenopus oocytes expressing hERG channels alone. D. Arithmetic means \pm SEM $(n=16-21)$ of the peak tail current as a function of voltage in Xenopus oocytes injected with water (white diamonds), with cRNA encoding PIKfyve alone (white squares), with cRNA encoding hERG alone (white circles) or with cRNA encoding hERG and PIKfyve (black circles). E. Arithmetic means \pm SEM ( $n=17-21$ ) of the normalized peak tail current as a function of voltage in Xenopus oocytes injected with cRNA encoding hERG alone (white circles) or with cRNA encoding hERG and PIKfyve (black circles).

the absolute current values were up-regulated by coexpression of wild type PIKfyve. Following normalization to the maximum peak tail current for each group, no significant differences were apparent between Xenopus oocytes expressing hERG together with PIKfyve and Xenopus oocytes expressing hERG alone. Along those lines, the voltage required for half maximal peak tail currents was similar in oocytes expressing hERG alone and in oocytes expressing hERG together with PIKfyve (Fig. 1E).

As PIKfyve is stimulated by protein kinase $\mathrm{PKB}$, additional experiments were performed to elucidate the effect of PKB on hERG current. As shown in Fig 2A,B, the current 


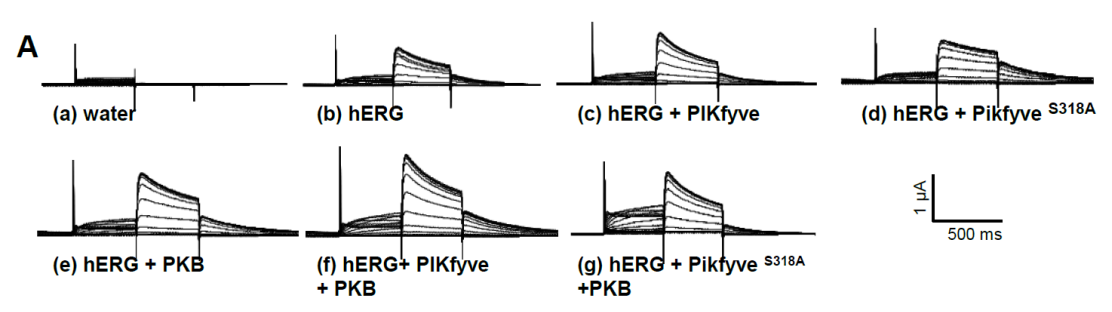

B

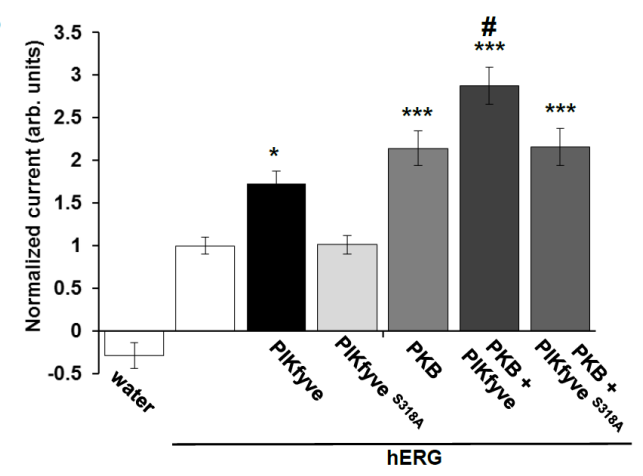

Fig. 2. Coexpression of PKB up-regulated hERG current, an effect augmented by PIKfyve but not by PKBresistant PIKfyve ${ }^{\mathrm{S} 318 \mathrm{~A}}$. A. Original tracings recorded in Xenopus oocytes injected with water (a), with cRNA encoding hERG alone (b), with cRNA encoding hERG and PIKfyve (c), with cRNA encoding hERG and PIKfyve $^{\mathrm{S} 318 \mathrm{~A}}$ (d), with cRNA encoding hERG and PKB (e), with cRNA encoding hERG and PIKfyve and PKB (f) or with cRNA encoding hERG, PKB and PIKfyve ${ }^{\mathrm{S318A}}(\mathrm{g})$. The oocytes were depolarized from $-80 \mathrm{mV}$ holding potential to different voltages followed by a $500 \mathrm{~ms}$ pulse to $-60 \mathrm{mV}$ evoking outward tail currents. B. Arithmetic means \pm SEM $(n=7-48)$ of the normalized outward tail current following a depolarization to $+70 \mathrm{mV}$ recorded in oocytes injected with water (water), with cRNA encoding hERG alone (hERG) with cRNA encoding hERG and PIKfyve (hERG+PIKfyve), with cRNA encoding hERG and PKB-resistant PIKfyve ${ }^{\mathrm{S} 318 \mathrm{~A}}$ (hERG+PIKfyve ${ }^{\mathrm{S} 18 \mathrm{~A}}$ ), with cRNA encoding hERG and protein kinase B (hERG + PKB), with cRNA encoding hERG, PKB and PIKfyve (hERG+PKB+PIKfyve) or with cRNA encoding hERG, PKB and PIKfyve ${ }^{\mathrm{s318A}}$ (hERG+P $\left.\mathrm{KB}+\mathrm{PIKfyve}^{\mathrm{S} 318 \mathrm{~A}}\right) .{ }^{*}(\mathrm{p}<0.05),{ }^{* * *}(\mathrm{p}<0.001)$ indicate statistically significant difference from Xenopus oocytes expressing hERG channels alone; \# $(\mathrm{p}<0.05)$ indicate statistically significant difference from Xenopus oocytes co-expressing hERG and PKB.

in hERG-expressing Xenopus oocytes was significantly increased by the coexpression of PKB. Additional expression of wild type PIKfyve was followed by a significant further increase of the current. In contrast, the additional co-expression of PKB with PKB resistant PIKfyve ${ }^{\mathrm{S3} 18 \mathrm{~A}}$ was not followed by a significant additional increase of the tail current.

The up-regulation of hERG activity following coexpression of PIKfyve could have resulted from an increase of channel protein abundance in the plasma membrane. Immunocytochemistry and confocal microscopy have been applied to visualize the hERG-HA channel protein abundance in the cell membrane. As illustrated in Fig. 3A, the co-expression of PIKfyve in hERG-HA expressing oocytes was followed by an increase of hERG protein abundance within the Xenopus oocyte cell membrane. In order to quantify hERG-HA protein abundance in the cell membrane of Xenopus oocytes, chemiluminescence was employed. As illustrated in Fig. 3B, the co-expression of PIKfyve was again followed by a significant increase of hERG-HA protein abundance within the Xenopus oocyte cell membrane.

The increase of hERG protein abundance in the cell membrane following coexpression of PIKfyve could have been due to enhanced channel protein stability due to delayed clearance of channel protein from the cell membrane. To determine the clearance of channel protein from the cell membrane, Xenopus oocytes expressing either hERG alone or together 
Fig. 3. Coexpression of PIKfyve increased hERG-HA protein abundance at the surface of hERG-expressing Xenopus oocytes. A. Confocal images of hERGHA protein cell surface expression in Xenopus oocytes injected with water (left panel), with cRNA encoding hERG-HA alone (middle panel) or with CRNA encoding hERGHA and PIKfyve
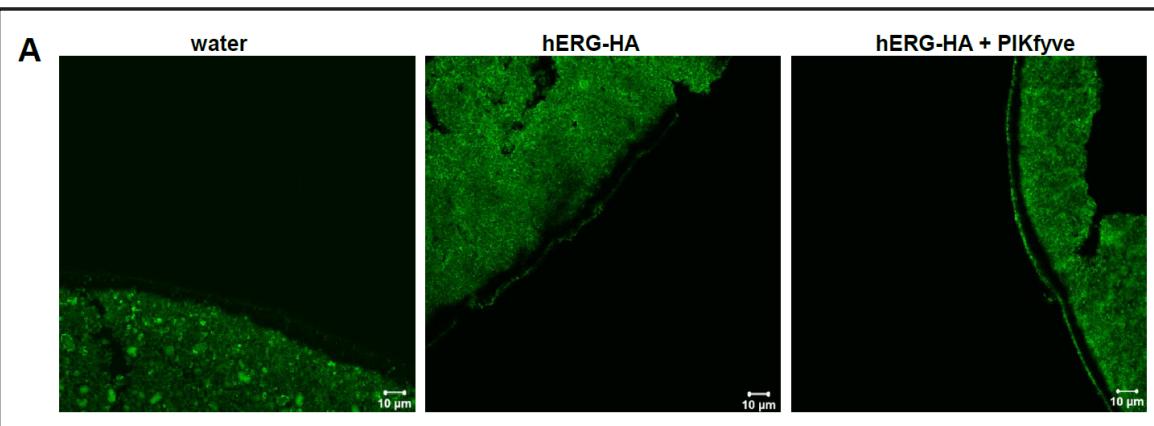

B

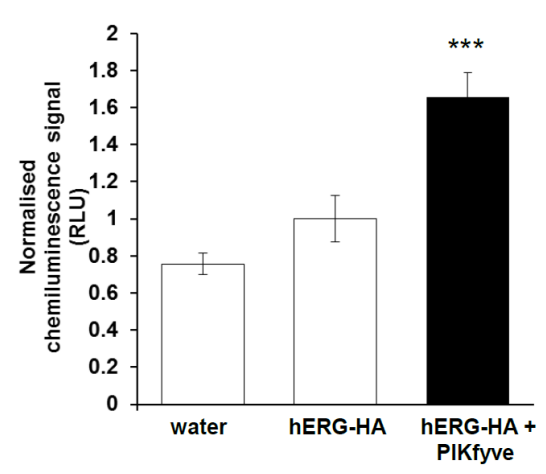

(right panel). B. Arithmetic means \pm SEM $(n=34-66)$ of hERG-HA protein abundance in the cell membrane measured by chemiluminescence in Xenopus oocytes injected with water (left bar), with cRNA encoding hERG-HA alone (middle bar), or cRNA encoding hERG-HA and wild type PIKfyve (right bar). ${ }^{* * *}(\mathrm{p}<0.001)$ indicates statistically significant difference from Xenopus oocytes expressing $\mathrm{hERG}$ channels alone.

with PIKfyve were treated with $5 \mu \mathrm{M}$ Brefeldin A, which blocks the insertion of new carrier protein into the cell membrane. As shown in Fig. 4, Brefeldin A treatment was followed by a decline of hERG current. The decline was similarly rapid in the oocytes coexpressing hERG and PIKfyve as in Xenopus oocytes expressing hERG alone.

\section{Discussion}

The present study identifies a novel mechanism of human ether-a-go-go (hERG) channel regulation. HERG channel protein abundance and thus hERG-mediated currents are up-regulated by phosphatidylinositol-3-phosphate-5-kinase (PIKfyve).

PIKfyve phosphorylates phosphatidylinositol 3-phosphate (PtdIns3P) leading to the production of phosphatidylinositol 3,5-bisphosphate $\left(\operatorname{PtdIns}(3,5) P_{2}\right)$ [21]. Phosphatidylinositides contribute to the regulation of membrane trafficking [24, 47-49]. PIKfyve presumably contributes to Rab-mediated endosomal transport. PIKfvye has been shown to associate with the Rab9 effector molecule p40 [50,51] leading to regulation of Rab9-mediated processes, such as transport from late endosome to the trans- Golgi network $[22,52]$. PIKfyve is phoshorylated by protein kinase B PKB/Akt leading to activation of the enzyme [11]. Treatment of cells with insulin is followed by PIKfyve phosphorylation, an effect depending on PI3-kinase-activity [11]. Phosphorylation of PIKfyve by PKB increases PtdIns $(3,5) P_{2}$ generation on the cytosolic surface of the PIKfyve containing intracellular vesicles and thus impacts on vesicle trafficking [11]. PIKfyve is localized in a subpopulation of highly dynamic vesicles containing the glucose carrier GLUT4 [11]. PIKfyve modifies the trafficking of those vesicles and thus participates in the regulation of GLUT4 trafficking [11].

In analogy hERG is similarly up-regulated by coexpression of PKB. Additional coexpression of wild type PIKfyve but not additional coexpression of the PKB-resistant mutant 


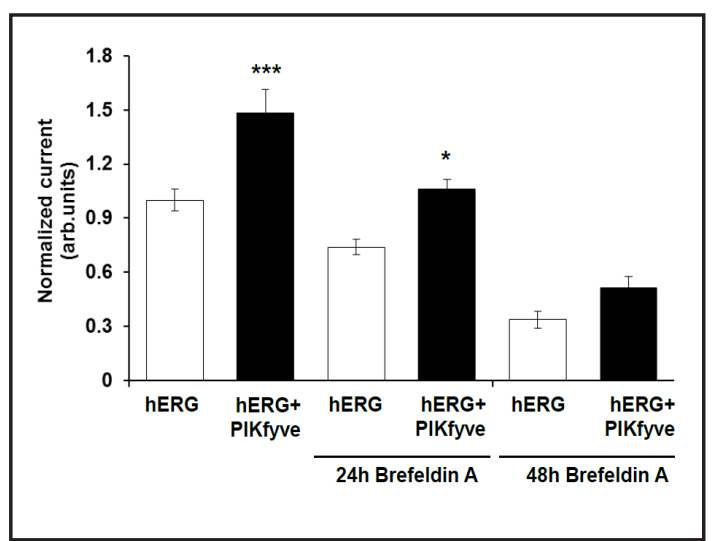

Fig. 4. Effects of Brefeldin A on Xenopus oocytes expressing hERG with or without PIKfyve. Arithmetic means \pm SEM $(n=27-33)$ of hERG current measured in Xenopus oocytes injected with cRNA encoding hERG without (white bars) and with (black bars) cRNA encoding PIKfyve and exposed to $5 \mu \mathrm{M}$ Brefeldin A for 0 hours, 24 hours or 48 hours prior to the measurements. ${ }^{*}(\mathrm{p}<0.05),{ }^{* * *}(\mathrm{p}<0.001)$ indicate statistically significant difference from Xenopus oocytes expressing hERG channels in the absence of PIKfyve.

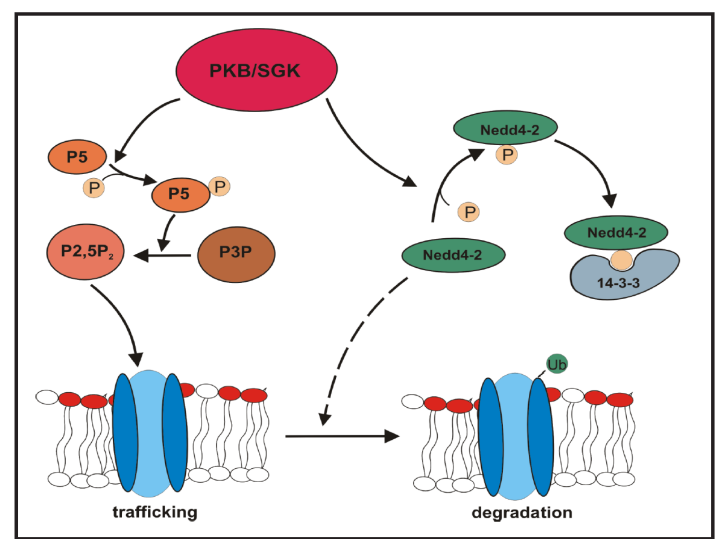

Fig. 5. Putative model illustrating the SGK/PKB dependent regulation of hERG by PIKfyve and Nedd4-2. PKB/Akt (PKB) and/or SGK isoforms phosphorylate ( $\mathrm{P}=$ phosphate) and thus activate PIKfyve (P5). PIKfyve in turn phosphorylates phosphatidylinositol 3-phosphate (P3P) leading to the production of phosphatidylinositol 3,5-bisphosphate $\left(\mathrm{P}_{3}, 5 \mathrm{P}_{2}\right)$, which in turn influences trafficking of channel bearing vesicles. SGK further phosphorylates Nedd4-2 thus fostering binding of Nedd4-2 to 14-3-3. Unphosphorylated Nedd4-2 ubiquitinates hERG thus preparing the channel for degradation.

PIKfyve $^{\mathrm{S318A}}$ augmented the effect of PKB, indicating that PKB is at least partially effective through phosphorylation of PIKfyve. The present observations did not address PIKfyve independent mechanisms involved in the effects of PKB/Akt or SGK isoforms on hERG channels. hERG has recently been shown to be regulated by the ubiquitin ligase Nedd4-2, which ubiquitinates target proteins thus preparing them for degradation $[9,53]$. SGK and PKB isoforms may phosphorylate Nedd4-2 leading to binding of the ubiquitin ligase to the scaffolding protein 14-3-3, which interferes with the interaction of Nedd4-2 with its target proteins $[54,55]$. Accordingly, the kinases could disrupt Nedd4-2-sensitive degradation of several ion channel proteins $[54,55]$ including hERG $[9,53]$.

SGK isoforms have further been shown to regulate expression of channel proteins by up-regulating the transcription factor NFKB [56]. However, to the best of our knowledge nothing is known about transcriptional regulation of hERG by NFKB.

The PIKfyve-sensitive regulation of hERG channels may be relevant for cardiac repolarization [1, 2]. Moreover, up-regulation of hERG channels by PIKfyve may be relevant for tumor cells, as hERG channel activity has been shown to participate in the regulation of tumor growth [3-5]. It should be pointed out, however, that regulation of channels by coexpressed signaling molecules in Xenopus oocytes does not allow predicting the impact of the respective signaling molecule on channel activity in the heart or in tumor cells. The expression level of the channel and the signaling molecule may be different in oocytes and any given mammalian cells. Moreover, the impact of the coexpressed signaling molecule on channel activity may be modified by additional signaling pathways expressed differently in Xenopus oocytes and mammalian cells. Nevertheless, the coexpression experiments may disclose interactions of signaling molecules with ion channels, which could be further tested in mammalian systems. Along those lines, the effect of SGK isoforms on ion channels prompted the analysis of QT intervals in individuals expressing a gain of function polymorphism of SGK1 in humans [57]. As a result, the polymorphism was indeed associated with a shortened QT interval, a finding underscoring the impact of SGK isoforms on repolarizing cardiac ion channels [57]. 


\section{Cellular Physiology Cell Physiol Biochem 2013;31:785-794 \begin{tabular}{l|l} 
and Biochemistry Published onIIne: IVlay 31, 2013 & $\begin{array}{l}\text { C 2013 S. Karger AG, Basel } \\
\text { www.karger.com/cpb }\end{array}$ \\
\hline
\end{tabular} \\ Pakladok/Almilaji/Munoz/Alesutan/Lang: PIKfyve-Regulated hERG Channels}

Lack of PIKfyve is apparently not compatible with survival, an observation highlighting the functional significance of PIKfyve-dependent regulation [58]. PIKfyve is obviously required for early embryonic development [58].

In conclusion, the present study demonstrates that PIKfyve is a powerful regulator of voltage-gated $\mathrm{K}^{+}$channel $\mathrm{hERG}$ and thus may participate in the shaping of the cardiac action potential and in the regulation of tumor growth.

\section{Acknowledgements}

The authors gratefully acknowledge the technical assistance by E. Faber and the meticulous preparation of the manuscript by A. Soleimanpour, L. Subasic and T. Loch. This work was supported by the DFG (GRK 1302/1, SE 1077/3 and SFB 773), and Open Access Publishing Fund of Tuebingen University.

\section{References}

1 Sanguinetti MC: Dysfunction of delayed rectifier potassium channels in an inherited cardiac arrhythmia. Ann N Y Acad Sci 1999;868:406-413.

2 Vincent GM: The molecular genetics of the long QT syndrome: genes causing fainting and sudden death. Annu Rev Med 1998;49:263-274.

-3 Arcangeli A: Expression and role of hERG channels in cancer cells. Novartis Found Symp 2005;266:225-232; discussion 232-224.

4 Asher V, Sowter H, Shaw R, Bali A, Khan R: Eag and HERG potassium channels as novel therapeutic targets in cancer. World J Surg Oncol 2010;8:113.

5 Pillozzi S, Arcangeli A: Physical and functional interaction between integrins and hERG1 channels in cancer cells. Adv Exp Med Biol 2010;674:55-67.

6 Glassmeier G, Hempel K, Wulfsen I, Bauer CK, Schumacher U, Schwarz JR: Inhibition of HERG1 K ${ }^{+}$channel protein expression decreases cell proliferation of human small cell lung cancer cells. Pflugers Arch 2012;463:365-376.

7 Raschi E, Vasina V, Poluzzi E, De Ponti F: The hERG K+ channel: target and antitarget strategies in drug development. Pharmacol Res 2008;57:181-195.

8 Maier G, Palmada M, Rajamanickam J, Shumilina E, Bohmer C, Lang F: Upregulation of HERG channels by the serum and glucocorticoid inducible kinase isoform SGK3. Cell Physiol Biochem 2006;18:177-186.

-9 Lamothe S, Zhang S: The Serum- and Glucocorticoid-Inducible Kinase SGK1 and SGK3 Regulate hERG Channel Expression via Ubiquitin Ligase Nedd4-2 and GTPase Rab11. J Biol Chem 2013;10.1074/jbc.M113.453670.

10 Seebohm G, Strutz-Seebohm N, Birkin R, Dell G, Bucci C, Spinosa MR, Baltaev R, Mack AF, Korniychuk G, Choudhury A, Marks D, Pagano RE, Attali B, Pfeufer A, Kass RS, Sanguinetti MC, Tavare JM, Lang F: Regulation of endocytic recycling of KCNQ1/KCNE1 potassium channels. Circ Res 2007;100:686-692.

11 Berwick DC, Dell GC, Welsh GI, Heesom KJ, Hers I, Fletcher LM, Cooke FT, Tavare JM: Protein kinase B phosphorylation of PIKfyve regulates the trafficking of GLUT4 vesicles. J Cell Sci 2004;117:5985-5993.

12 Ikonomov OC, Sbrissa D, Shisheva A: YM201636, an inhibitor of retroviral budding and PIKfyve-catalyzed PtdIns(3,5)P2 synthesis, halts glucose entry by insulin in adipocytes. Biochem Biophys Res Commun 2009;382:566-570.

13 Shojaiefard M, Strutz-Seebohm N, Tavare JM, Seebohm G, Lang F: Regulation of the $\mathrm{Na}^{+}$, glucose cotransporter by PIKfyve and the serum and glucocorticoid inducible kinase SGK1. Biochem Biophys Res Commun 2007;359:843-847.

14 Bogatikov E, Munoz C, Pakladok T, Alesutan I, Shojaiefard M, Seebohm G, Foller M, Palmada M, Bohmer C, Broer S, Lang F: Up-Regulation of Amino Acid Transporter SLC6A19 Activity and Surface Protein Abundance by PKB/ Akt and PIKfyve. Cell Physiol Biochem 2012;30:1538-1546.

15 Michell RH, Heath VL, Lemmon MA, Dove SK: Phosphatidylinositol 3,5-bisphosphate: metabolism and cellular functions. Trends Biochem Sci 2006;31:52-63. 


\section{Cellular Physiology Cell Physiol Biochem 2013;31:785-794

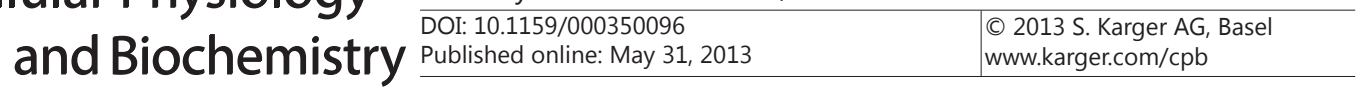 \\ Pakladok/Almilaji/Munoz/Alesutan/Lang: PIKfyve-Regulated hERG Channels}

16 Bazenet CE, Ruano AR, Brockman JL, Anderson RA: The human erythrocyte contains two forms of phosphatidylinositol-4-phosphate 5-kinase which are differentially active toward membranes. J Biol Chem 1990;265:18012-18022.

17 Cabezas A, Pattni K, Stenmark H: Cloning and subcellular localization of a human phosphatidylinositol 3-phosphate 5-kinase, PIKfyve/Fab1. Gene 2006;371:34-41.

18 Gillooly DJ, Morrow IC, Lindsay M, Gould R, Bryant NJ, Gaullier JM, Parton RG, Stenmark H: Localization of phosphatidylinositol 3-phosphate in yeast and mammalian cells. EMBO J 2000;19:4577-4588.

19 Shisheva A: PIKfyve: Partners, significance, debates and paradoxes. Cell Biol Int 2008;32:591-604.

20 Sbrissa D, Ikonomov OC, Shisheva A: Phosphatidylinositol 3-phosphate-interacting domains in PIKfyve. Binding specificity and role in PIKfyve. Endomenbrane localization. J Biol Chem 2002;277:6073-6079.

-21 Sbrissa D, Ikonomov OC, Shisheva A: PIKfyve, a mammalian ortholog of yeast Fab1p lipid kinase, synthesizes 5-phosphoinositides. Effect of insulin. J Biol Chem 1999;274:21589-21597.

22 Rutherford AC, Traer C, Wassmer T, Pattni K, Bujny MV, Carlton JG, Stenmark H, Cullen PJ: The mammalian phosphatidylinositol 3-phosphate 5-kinase (PIKfyve) regulates endosome-to-TGN retrograde transport. J Cell Sci 2006;119:3944-3957.

23 Botelho RJ: Changing phosphoinositides "on the fly": how trafficking vesicles avoid an identity crisis. Bioessays 2009;31:1127-1136.

24 de Lartigue J, Polson H, Feldman M, Shokat K, Tooze SA, Urbe S, Clague MJ: PIKfyve regulation of endosomelinked pathways. Traffic 2009;10:883-893.

25 Rusten TE, Rodahl LM, Pattni K, Englund C, Samakovlis C, Dove S, Brech A, Stenmark H: Fab1 phosphatidylinositol 3-phosphate 5-kinase controls trafficking but not silencing of endocytosed receptors. Mol Biol Cell 2006;17:3989-4001.

26 Ikonomov OC, Fligger J, Sbrissa D, Dondapati R, Mlak K, Deeb R, Shisheva A: Kinesin adapter JLP links PIKfyve to microtubule-based endosome-to-trans-Golgi network traffic of furin. J Biol Chem 2009;284:3750-3761.

27 Strutz-Seebohm N, Shojaiefard M, Christie D, Tavare J, Seebohm G, Lang F: PIKfyve in the SGK1 mediated regulation of the creatine transporter SLC6A8. Cell Physiol Biochem 2007;20:729-734.

-28 Alesutan IS, Ureche ON, Laufer J, Klaus F, Zurn A, Lindner R, Strutz-Seebohm N, Tavare JM, Boehmer C, Palmada M, Lang UE, Seebohm G, Lang F: Regulation of the glutamate transporter EAAT4 by PIKfyve. Cell Physiol Biochem 2010;25:187-194.

29 Gehring EM, Zurn A, Klaus F, Laufer J, Sopjani M, Lindner R, Strutz-Seebohm N, Tavare JM, Boehmer C, Palmada M, Lang UE, Seebohm G, Lang F: Regulation of the glutamate transporter EAAT2 by PIKfyve. Cell Physiol Biochem 2009;24:361-368.

-30 Klaus F, Gehring EM, Zurn A, Laufer J, Lindner R, Strutz-Seebohm N, Tavare JM, Rothstein JD, Boehmer C, Palmada M, Gruner I, Lang UE, Seebohm G, Lang F: Regulation of the $\mathrm{Na}^{+}$-coupled glutamate transporter EAAT3 by PIKfyve. Neurochem Int 2009;54:372-377.

-31 Seebohm G, Strutz-Seebohm N, Ursu ON, Preisig-Muller R, Zuzarte M, Hill EV, Kienitz MC, Bendahhou S, Fauler M, Tapken D, Decher N, Collins A, Jurkat-Rott K, Steinmeyer K, Lehmann-Horn F, Daut J, Tavare JM, Pott L, Bloch W, Lang F: Altered stress stimulation of inward rectifier potassium channels in Andersen-Tawil syndrome. FASEB J 2012;26:513-522.

-32 Sopjani M, Kunert A, Czarkowski K, Klaus F, Laufer J, Foller M, Lang F: Regulation of the Ca ${ }^{2+}$ channel TRPV6 by the kinases SGK1, PKB/Akt, and PIKfyve. J Membr Biol 2010;233:35-41.

-33 Gehring EM, Lam RS, Siraskar G, Koutsouki E, Seebohm G, Ureche ON, Ureche L, Baltaev R, Tavare JM, Lang F: PIKfyve upregulates CFTR activity. Biochem Biophys Res Commun 2009;390:952-957.

-34 Seebohm G, Neumann S, Theiss C, Novkovic T, Hill EV, Tavare JM, Lang F, Hollmann M, Manahan-Vaughan D, Strutz-Seebohm N: Identification of a novel signaling pathway and its relevance for GluA1 recycling. PLoS One 2012;7:e33889.

-35 Mohamed MR, Alesutan I, Foller M, Sopjani M, Bress A, Baur M, Salama RHM, Bakr MS, Mohamed MA, Blin N, Lang F, Pfister M: Functional Analysis of a Novel I71N Mutation in the GJB2 Gene Among Southern Egyptians Causing Autosomal Recessive Hearing Loss. Cell Physiol Biochem 2010;26:959-966.

-36 Huang FD, Chen J, Lin M, Keating MT, Sanguinetti MC: Long-QT syndrome-associated missense mutations in the pore helix of the HERG potassium channel. Circulation 2001;104:1071-1075.

-37 Rexhepaj R, Dermaku-Sopjani M, Gehring EM, Sopjani M, Kempe DS, Foller M, Lang F: Stimulation of electrogenic glucose transport by glycogen synthase kinase 3. Cell Physiol Biochem 2010;26:641-646. 


\section{Cellular Physiology Cell Physiol Biochem 2013;31:785-794 \begin{tabular}{ll|l} 
and Biochemistry & $\begin{array}{l}\text { DOI 10.1159/000350096 } \\
\text { Published online: May 31, } 2013\end{array}$ & $\begin{array}{l}\text { O 2013 S. Karger AG, Basel } \\
\text { www.karger.com/cpb }\end{array}$ \\
\cline { 2 - 3 }
\end{tabular} \\ Pakladok/Almilaji/Munoz/Alesutan/Lang: PIKfyve-Regulated hERG Channels}

-38 Kempe DS, Ackermann TF, Boini KM, Klaus F, Umbach AT, Dermaku-Sopjani M, Judenhofer MS, Pichler BJ, Capuano P, Stange G, Wagner CA, Birnbaum MJ, Pearce D, Foller M, Lang F: Akt2/PKBbeta-sensitive regulation of renal phosphate transport. Acta Physiol (Oxf) 2010;200:75-85.

-39 Henrion U, Zumhagen S, Steinke K, Strutz-Seebohm N, Stallmeyer B, Lang F, Schulze-Bahr E, Seebohm G: Overlapping cardiac phenotype associated with a familial mutation in the voltage sensor of the KCNQ1 channel. Cell Physiol Biochem 2012;29:809-818.

40 Hosseinzadeh Z, Bhavsar SK, Lang F: Downregulation of ClC-2 by JAK2. Cell Physiol Biochem 2012;29:737-742.

41 Alesutan I, Sopjani M, Dermaku-Sopjani M, Munoz C, Voelkl J, Lang F: Upregulation of Na-coupled glucose transporter SGLT1 by Tau tubulin kinase 2. Cell Physiol Biochem 2012;30:458-465.

-42 Pathare G, Foller M, Daryadel A, Mutig K, Bogatikov E, Fajol A, Almilaji A, Michael D, Stange G, Voelkl J, Wagner CA, Bachmann S, Lang F: OSR1-Sensitive Renal Tubular Phosphate Reabsorption. Kidney Blood Press Res 2012;36:149-161.

-43 Strutz-Seebohm N, Pusch M, Wolf S, Stoll R, Tapken D, Gerwert K, Attali B, Seebohm G: Structural basis of slow activation gating in the cardiac I Ks channel complex. Cell Physiol Biochem 2011;27:443-452.

44 Hosseinzadeh Z, Bhavsar SK, Sopjani M, Alesutan I, Saxena A, Dermaku-Sopjani M, Lang F: Regulation of the Glutamate Transporters by JAK2. Cell Physiol Biochem 2011;28:693-702.

45 Nebenfuhr A, Ritzenthaler C, Robinson DG: Brefeldin A: deciphering an enigmatic inhibitor of secretion. Plant Physiol 2002;130:1102-1108.

-46 Dermaku-Sopjani M, Sopjani M, Saxena A, Shojaiefard M, Bogatikov E, Alesutan I, Eichenmuller M, Lang F: Downregulation of NaPi-IIa and NaPi-IIb $\mathrm{Na}^{+}$-coupled Phosphate Transporters by Coexpression of Klotho. Cell Physiol Biochem 2011;28:251-258.

-47 Zheng Q Bobich JA: ADP-ribosylation factor6 regulates both [3H]-noradrenaline and [14C]-glutamate exocytosis through phosphatidylinositol 4,5-bisphosphate. Neurochem Int 2004;45:633-640.

-48 Morris AJ: Regulation of phospholipase D activity, membrane targeting and intracellular trafficking by phosphoinositides. Biochem Soc Symp 2007;74:247-257.

-49 Ikonomov OC, Sbrissa D, Fenner H, Shisheva A: PIKfyve-ArPIKfyve-Sac3 core complex: contact sites and their consequence for Sac3 phosphatase activity and endocytic membrane homeostasis. J Biol Chem 2009;284:35794-35806.

-50 Sbrissa D, Ikonomov OC, Shisheva A: Analysis of potential binding of the recombinant Rab9 effector p40 to phosphoinositide-enriched synthetic liposomes. Methods Enzymol 2005;403:696-705.

51 Ikonomov OC, Sbrissa D, Mlak K, Deeb R, Fligger J, Soans A, Finley RL Jr, Shisheva A: Active PIKfyve associates with and promotes the membrane attachment of the late endosome-to-trans-Golgi network transport factor Rab9 effector p40. J Biol Chem 2003;278:50863-50871.

52 Lombardi D, Soldati T, Riederer MA, Goda Y, Zerial M, Pfeffer SR: Rab9 functions in transport between late endosomes and the trans Golgi network. EMBO J 1993;12:677-682.

-53 Guo J, Wang T, Li X, Shallow H, Yang T, Li W, Xu J, Fridman MD, Yang X, Zhang S: Cell surface expression of human ether-a-go-go-related gene (hERG) channels is regulated by caveolin-3 protein via the ubiquitin ligase Nedd4-2. J Biol Chem 2012;287:33132-33141.

-54 Lang F, Bohmer C, Palmada M, Seebohm G, Strutz-Seebohm N, Vallon V: (Patho)physiological significance of the serum- and glucocorticoid-inducible kinase isoforms. Physiol Rev 2006;86:1151-1178.

-55 Yang B, Kumar S: Nedd4 and Nedd4-2: closely related ubiquitin-protein ligases with distinct physiological functions. Cell Death Differ 2010;17:68-77.

56 Lang F, Eylenstein A, Shumilina E: Regulation of Orai1/STIM1 by the kinases SGK1 and AMPK. Cell Calcium 2012;52:347-354.

57 Busjahn A, Seebohm G, Maier G, Toliat MR, Nurnberg P, Aydin A, Luft FC, Lang F: Association of the serum and glucocorticoid regulated kinase (sgk1) gene with QT interval. Cell Physiol Biochem 2004;14:135-142.

58 Ikonomov OC, Sbrissa D, Delvecchio K, Xie Y, Jin JP, Rappolee D, Shisheva A: The phosphoinositide kinase PIKfyve is vital in early embryonic development: preimplantation lethality of PIKfyve $\mathrm{e}^{-/}$embryos but normality of PIKfyve ${ }^{+/}$mice. J Biol Chem 2011;286:13404-13413. 